code number:

\title{
Probing interface magnetism in the FeMn/NiFe exchange bias system using magnetic second harmonic generation
}

\author{
Luiz C. Sampaio, Al Alexandra Mougin and Jacques Ferré \\ Laboratoire de Physique des Solides, UMR CNRS 8502, \\ Bât. 510, Université Paris Sud, 91405 Orsay, France \\ Patrick Georges and Alain Brun \\ Laboratoire Charles Fabry de l'Institut d'Optique Théorique et Appliquée (IOTA), \\ Bât. 503, UMR CNRS 8501, Université Paris Sud, 91405 Orsay, France \\ Harry Bernas \\ Centre de Spectrométrie Nucléaire et de Spectrométrie de Masse, \\ UMR CNRS 8609, Bât. 108, Université Paris Sud, 91405 Orsay, France

\begin{abstract}
Stefan Poppe, Tim Mewes, Jürgen Fassbender, and Burkard Hillebrands
Fachbereich Physik and Forschungs- und Entwicklungsschwerpunkt Materialwissenschaften, Erwin-Schrödinger-Straße 56, 67663 Kaiserslautern, Germany
\end{abstract}

(Dated: November 12, 2018)

\begin{abstract}
Second harmonic generation magneto-optic Kerr effect (SHMOKE) experiments, sensitive to buried interfaces, were performed on a polycrystalline NiFe/FeMn bilayer in which areas with different exchange bias fields were prepared using $5 \mathrm{KeV} \mathrm{He}$ ion irradiation. Both reversible and irreversible uncompensated spins are found in the antiferromagnetic layer close to the interface with the ferromagnetic layer. The SHMOKE hysteresis loop shows the same exchange bias field as obtained from standard magnetometry. We demonstrate that the exchange bias effect is controlled by pinned uncompensated spins in the antiferromagnetic layer.

PACS numbers: 33.55.Fi, 75.70.-i, 42.65.-k, 75.30.Gw
\end{abstract}

The magnetic exchange interaction between an antiferromagnetic $(\mathrm{AF})$ and an adjacent ferromagnetic $(\mathrm{F})$ layer may lead to the exchange bias effect discovered in 1956 [1, 2]. Among other various intriguing features, this effect leads to a shift of the $\mathrm{F}$ hysteresis loop along the field axis by the so-called exchange bias field $H_{e b}$. For recent reviews see Refs. 3, 4, 5. Proposed models to account for the exchange bias involve (i) domain walls or partial domain walls in the AF layer which are either parallel [5, 6] or perpendicular [7] to the interface, and/or (ii) uncompensated AF layer magnetic moments at the interface [7, 8, 9] and/or in the bulk [9, 10]. In most exchange bias models, the interfacial uncompensated spins are linked to roughness, structural defects, or disoriented grains. Although uncompensated spins have been already evidenced [11, their behavior during the F layer magnetic reversal has not been reported so far and, experimentally, the relationship between uncompensated spins and exchange bias is still unclear. In the special case where artificial random defects can be introduced in the AF layer (such as in a diluted antiferromagnet), the so-called "domain state model" [9, 10] showed that the exchange bias effect stems from the volume AF spin arrangement triggered by non magnetic defects. In this model, AF interfacial reversible and irreversible uncompensated spins (creating $M_{r e v}^{F / A F}$ and $M_{i r r}^{A F}$ respectively) are distinguished. Some of the interfacial AF uncompensated spins reverse under the action of an external mag- netic field and the additional effective interface exchange field originating from the magnetized $\mathrm{F}$ layer, whereas the rest of the AF uncompensated spins remain frozen in the same range of applied fields. The reversible uncompensated spins hysteresis loop is found to be shifted along the field axis by $H_{e b}$ and along the magnetization axis by an amount directly proportional to $M_{i r r}^{A F}$, which scales with $H_{e b}$ [10]. Using superconducting quantum interference device magnetometry, this vertical shift of the hysteresis loop of F/AF bilayers has already been measured and related to the exchange bias field sign [12], although its origin was not determined.

Here we study the second-harmonic magneto-optic Kerr effect (SHMOKE) in an exchange-bias system. A second-harmonic signal in centrosymmetric materials is selectively generated at their interfaces due to symmetry breaking, so that the effect only senses about 2 monolayers in the vicinity of flat surfaces / interfaces [13, 14]. In contrast, the standard linear MOKE signal originates mainly from the bulk of the F layers. Generally, the second harmonic optical polarization $\vec{P}(2 \omega)$, generated at a single interface, consists of both magnetic and nonmagnetic contributions due to the magnetic optical susceptibility $\chi_{m}(2 \omega)$ (linear with respect to the magnetization $\vec{M})$ and the non-magnetic one $\chi_{n m}(2 \omega)$ (indepen- 
dent or even with respect to $\vec{M}$ ),

$$
\begin{aligned}
P_{i}(2 \omega) & =\sum_{j, k} \chi_{i j k}(2 \omega) E_{j}(\omega) E_{k}(\omega) \\
\chi(2 \omega, \vec{M}) & =\chi_{n m}(2 \omega, \vec{M})+\chi_{m}(2 \omega, \vec{M}) \\
\chi(2 \omega,-\vec{M}) & =\chi_{n m}(2 \omega, \vec{M})-\chi_{m}(2 \omega, \vec{M})
\end{aligned}
$$

where $E_{j}(\omega)$ are the electric field components of the incident light and $\chi_{i j k}(2 \omega)$ the second harmonic susceptibility tensor elements. Each surface or interface contributes to the SHMOKE signal so that the measured intensity from a $n$ multilayer is given by the sum of all interfering signals: $I(2 \omega) \propto \sum_{(a, b)=(1, \ldots, n)} P_{a}(2 \omega) P_{b}^{*}(2 \omega)$.

In the present study, polycrystalline bilayers of ferromagnetic $\mathrm{Ni}_{81} \mathrm{Fe}_{19}$ and antiferromagnetic $\mathrm{Fe}_{50} \mathrm{Mn}_{50}$ were used in order to tailor the exchange bias field by light ion irradiation [15, 16]. Bilayers of $10 \mathrm{~nm} \mathrm{FeMn}$ and $5 \mathrm{~nm}$ $\mathrm{NiFe}$ were evaporated on a $15 \mathrm{~nm}$ thick $\mathrm{Cu}$ buffer layer deposited on a thermally oxidized Si substrate. A thin $\mathrm{Cr}$ cover layer protected the samples from oxidation. In order to initialize the unidirectional anisotropy, the samples were heated and then field cooled. This led to a homogeneous exchange bias field $H_{e b}$ of 200 Oe across the sample, as determined from linear MOKE (see Fig. 1a). After preparation, the exchange bias was modified in different areas of the sample using $5 \mathrm{keV}$ He ion irradiation in a fluence range between $9 \cdot 10^{13}$ and $2 \cdot 10^{16}$ ions $/ \mathrm{cm}^{2}$, leading to $H_{e b}$ values between 100 Oe and 350 Oe (see Fig. 2a, full symbols), consistent with previous work [16]. A discussion of the exchange bias field evolution with ion fluence is given in Ref. [16]. Based upon the latter and ion stopping calculations 117 we note that the interface roughness is not affected when ion fluences are below a few times $10^{16}$ ions $/ \mathrm{cm}^{2}$.

SHMOKE experiments were performed with a modelocked Ti:Sapphire laser operating at a central wavelength of $800 \mathrm{~nm}$, emitting light pulses of width $100 \mathrm{fs}$ at a repetition rate of $86 \mathrm{MHz}$. All experiments were performed in reflection; the average power at the sample surface was $50 \mathrm{~mW}$ within a focus of $30-40 \mu \mathrm{m}$. Using a $P$ polarized laser beam $\left(P_{i n}\right)$ at an incident angle of $45^{\circ}$ the $\mathrm{SH}$ signal can be measured in the transverse configuration $(T)$, and in the longitudinal one $(L)$ when combined with a polarization analysis. The complex effective magnetic and non-magnetic susceptibilities $\chi_{m}$ and $\chi_{n m}$ which enter the calculation of the SHMOKE intensity are functions of $\chi_{i j k}(2 \omega)$ tensor elements depending on the measurement geometry, hence they differ in the transverse or longitudinal configurations [13]. In order to extract information on the magnetization at the $\mathrm{F} / \mathrm{AF}$ interface, the $\mathrm{SH}$ contribution of each individual interface and surface $(\mathrm{Cu} / \mathrm{FeMn}, \mathrm{FeMn} / \mathrm{NiFe}, \mathrm{NiFe} / \mathrm{Cr}$ and $\mathrm{Cr} /$ air $)$ must be analyzed. Due to the same crystallographic structure and the close chemical nature of the FeMn and NiFe lay-

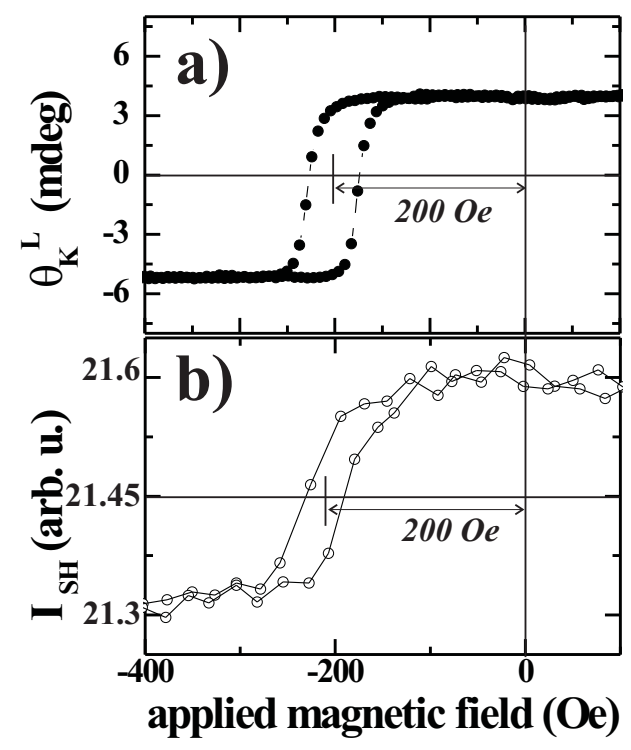

FIG. 1: Bulk and interfacial magnetization reversal loops investigated by linear MOKE (a), and second harmonic MOKE (b), respectively. $\theta_{K}^{L}$ is the linear Kerr rotation and $I_{S H}$ is the SHMOKE intensity in the transverse configuration.

ers, independent of the measurement configuration, $\chi_{n m}$ originating from the $\mathrm{FeMn} / \mathrm{NiFe}$ interface has a smaller value than those of the upper $\mathrm{NiFe} / \mathrm{Cr}$ and $\mathrm{Cr} /$ air interfaces. Therefore (see definition of $R_{L}=\left|\frac{\chi_{m}}{\chi_{n m}}\right|_{L}$ in Eq. (3b ) ), most of the magnetic SH signal originates from the $\mathrm{FeMn} / \mathrm{NiFe}$ interface whose $R_{L}$ is large. No signal is expected from the bulk centrosymmetric $\mathrm{F}$ or AF layers 13.

In transverse geometry, with $P$-polarized incident light, the SH outgoing beam is still $P$-polarized. Thus, the second harmonic intensity was measured as a function of the applied field, which was oriented perpendicular to the plane of incidence and parallel to the exchange bias direction. The resulting hysteresis loop is shifted along the field axis by the same bias as that of the bulk F layer (Figure 1 b). We conclude that SHMOKE selectively probes reversible $\left(M_{r e v}^{F / A F}\right)$ uncompensated spins at the interface, which are coupled to the F layer [9, 10 . Moreover, due to some frustration at the F/AF interface, the SHMOKE hysteresis loop is broader than its bulk F layer counterpart. As shown in Fig. 2a, at all fluences the exchange bias field values measured via SH or linear MOKE agree within the error bars.

Another magnetization term $\left(M_{i r r}^{A F}\right)$, required to induce any $H_{e b}$ and which remains unaffected by the $\mathrm{F}$ layer reversal (between $\pm H$, with $|H|=H_{\text {sat }}^{F}$ ), has been probed as follows. In order to separate out the magnetic and non magnetic contributions (see Eq. (1)), the ion fluence dependence of the non-magnetic contribution 


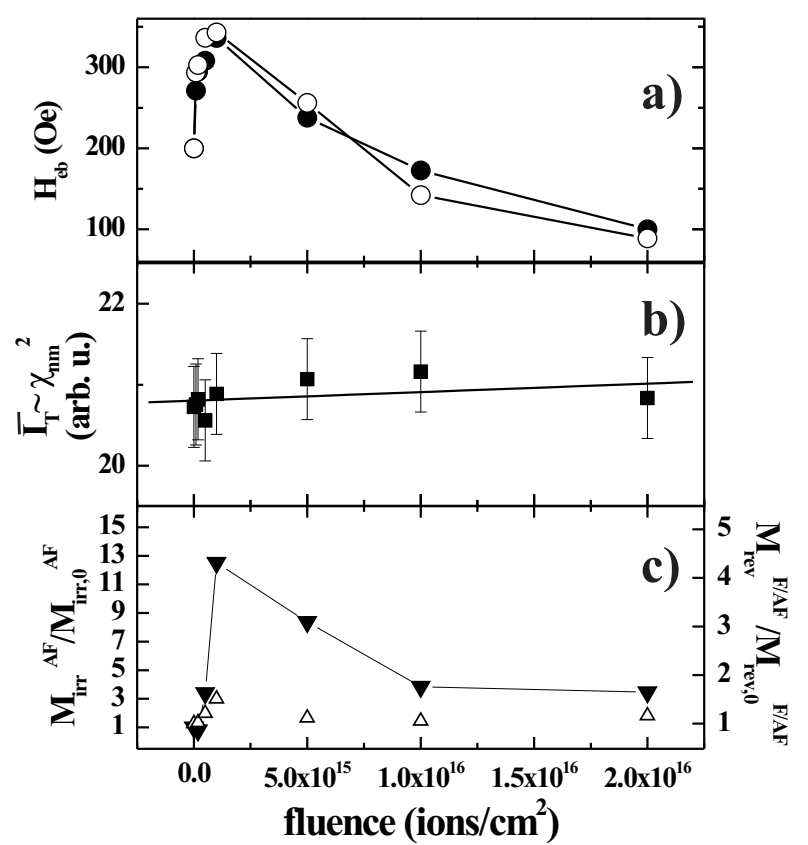

FIG. 2: Ion fluence dependence of: a) exchange bias field (full symbols: MOKE, open symbols: SHMOKE), b) nonmagnetic contribution to the SHMOKE signal $\left(\overline{I_{T}}\right)$, and c) irreversible $\left(M_{i r r}^{A F} / M_{i r r, 0}^{A F}\right.$, full triangles) and reversible magnetization $\left(M_{r e v}^{F / A F} / M_{r e v, 0}^{F / A F}\right.$, empty triangles $)$, normalized to their initial value and deduced from asymmetry measurement in the longitudinal configuration. Solid lines are guides to the eye.

was first determined. Commonly [18] since the magnetic term is small as compared to the average part of the $\mathrm{SH}$ intensity 19], the non-magnetic contribution scales with the average transverse SHMOKE intensity $\overline{I_{T}}$ [20]:

$$
\begin{aligned}
\overline{I_{T}} & =\frac{I_{T}(2 \omega,+H)+I_{T}(2 \omega,-H)}{2} \\
& \propto\left|\alpha \chi_{n m}\right|_{T}^{2}+\left|\beta \chi_{m}\right|_{T}^{2} \approx\left|\alpha \chi_{n m}\right|_{T}^{2}
\end{aligned}
$$

$\alpha$ and $\beta$ are the effective Fresnel factors [20]. $\overline{I_{T}}$ (and thus the effective non-magnetic optical susceptibility $\left.\left|\chi_{n m}^{2}\right|_{T}\right)$ is shown in Fig. 2 $b$ as a function of ion fluence. Practically no evolution is observed, confirming that no significant interface broadening occurs upon irradiation in this fluence range.

Polarization measurements were performed in the longitudinal configuration, and the magnetic contributions to the $\mathrm{SH}$ signal were extracted from the asymmetry $A_{L}(H, \psi)$, defined as the normalized intensity difference when the $\mathrm{F}$ magnetization is reversed 13. At the F/AF interface, the structural $\left(\chi_{n m}\right)$ and two distinct magnetic $\left(\chi_{m}\left(M_{r e v}^{F / A F}\right)\right.$ and $\left.\chi_{m}\left(M_{i r r}^{A F}\right)\right)$ contributions can give rise to a second harmonic signal. Each magnetic contribution induces a rotation of the polarization of the outgoing beam (from $P$ to $S$ ) [13] and is phase shifted relative to the structural term: $\varphi_{\text {rev }}$ (respectively $\varphi_{\text {irr }}$ ) is the phase angle between $\chi_{m}\left(M_{r e v}^{F / A F}\right)$ (respectively $\left.\chi_{m}\left(M_{i r r}^{A F}\right)\right)$ and $\chi_{n m}$. Usually, when the applied field is reversed, all magnetic contributions (linear with $\mathrm{M}$ ) to the second harmonic polarization change sign. Here, between $\pm H$, we assume that $M_{\text {rev }}^{F / A F}$ reverses i.e. $\chi_{m}\left(M_{r e v}^{F / A F}\right)$ changes its sign whereas $M_{i r r}^{A F}$ is pinned i.e. $\chi_{m}\left(M_{i r r}^{A F}\right)$ does not change. This leads to the following expression of $A_{L}$ :

$$
\begin{gathered}
A_{L}(H, \psi)=\frac{I(2 \omega,+H)-I(2 \omega,-H)}{I(2 \omega,+H)+I(2 \omega,-H)} \\
=\frac{2 R_{L}^{r e v} R_{L}^{i r r} \cos \left(\varphi_{r e v}-\varphi_{i r r}\right) \tan ^{2} \psi+2 R_{L}^{r e v} \tan \psi \cdot \cos \varphi_{r e v}}{1+\left(R_{L}^{r e v 2}+R_{L}^{i r r 2}\right) \tan ^{2} \psi+2 R_{L}^{i r r} \tan \psi \cdot \cos \varphi_{i r r}}
\end{gathered}
$$

where $\psi$ is the analyzer angle and $R_{L}^{r e v}=\left|\frac{\chi_{m}\left(M_{r e v}^{F / A F}\right)}{\chi_{n m}}\right|_{L}$ (correspondingly, $R_{L}^{i r r}=\left|\frac{\chi_{m}\left(M_{i r r}^{A F}\right)}{\chi_{n m} m}\right|_{L}$ ). Experimentally, for each analyser angle $\psi$, the $\mathrm{SH}$ intensity is measured upon reversal of the $\mathrm{F}$ layer. This enables us to determine $A_{L}(\psi)$. Examples are shown in Fig. 3 .

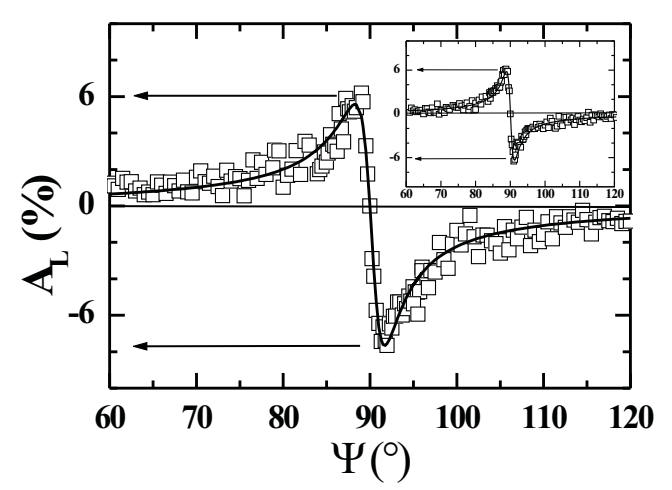

FIG. 3: SHMOKE asymmetry $A_{L}$ in longitudinal geometry as a function of the analyzer angle $\psi$ at a fluence of $10^{15} \mathrm{He}$ ions $/ \mathrm{cm}^{2}\left(10^{16} \mathrm{He}\right.$ ions $/ \mathrm{cm}^{2}$ in the inset). The solid line is the fit described in the text.

Due to the irreversible term and according to Eq. (3b), two distinct absolute extrema values are evidenced. The difference between them depends on the bias field (it is lower for smaller biases as shown in the inset). By fitting the asymmetry $A_{L}$ with Eq. (3b), $R_{L}^{\text {rev }}$ and $R_{L}^{\text {irr }}$ are obtained. Since the effective non-magnetic contribution to the second-harmonic generation signal is constant [21, $\left|\chi_{m}\left(M_{i r r}^{A F}\right) / \chi_{n m}\right|_{L}$ (respectively $\left.\left|\chi_{m}\left(M_{r e v}^{F / A F}\right) / \chi_{n m}\right|_{L}\right)$ is directly proportional to $M_{i r r}^{A F}$ (respectively $M_{r e v}^{F / A F}$ ) along the magnetic field direction 13].

Our key experimental results (Fig. 2 $c$ ) are as follows: i) the ion fluence dependence of the irreversible uncompensated spins (full triangles) reproduces that of the exchange bias field (Fig. 2a), ii) a 13-fold increase in the irreversible magnetization and the one order of 
magnitude smaller interfacial magnetic component enhancement (empty triangles) result in a bias field enhancement by a factor of 1.8 , iii) the proportion of irreversible (compared to reversible) spins given by the ratio $\chi_{m}\left(M_{i r r}^{A F}\right) / \chi_{m}\left(M_{r e v}^{F / A F}\right)$ can reach about $10 \%$. In the same fluence regime, the linear Kerr rotation due to the bulk $\mathrm{F}$ layer is constant [16]. The fluence behavior of $M_{i r r}^{A F}$ and $H_{e b}$ indicates that pinned uncompensated spins in the AF layer control the bias field. Moreover, this rigid $\mathrm{AF}$ moment is much smaller than the reversible magnetization (maximum ca. $10 \%$ ). According to the domain state model [9, 10], the exchange bias field is $H_{e b}=J_{i n t} M_{i r r}^{A F} / \mu t$, with $J_{i n t}$ the interface coupling, $\mu$ the magnetic moment per atom and $t$ the $\mathrm{F}$ layer thickness. Random exchange defects or antisites are formed in the AF layer via diluting impurities or irradiation. Under the action of the exchange field originating from the magnetized $\mathrm{F}$ layer, the AF domain structure is triggered and the uncompensated spins associated to such defects exhibit an excess magnetization. The latter is presumably reversible in the vicinity of the $\mathrm{F}$ layer and pinned if deeper in the AF layer. In the domain state model, both interface coupling and magnetic moment are assumed to be fixed and dilution only drives $M_{i r r}^{A F}$. In our experimental findings, additional information regarding the interfacial contribution is obtained; changes in local chemical order due to irradiation lead to a slight evolution of the interfacial reversible magnetization. This mixing effect counteracts the increase of $M_{i r r}^{A F}$ and - for fluences larger than those investigated here - reduces the bias as reported in Ref 16$]$.

To obtain insight on the exchange bias mechanism, we have combined ion irradiation-induced tuning of the irreversible magnetization in the AF layer with interface-selective second-harmonic magneto-optic Kerr detection. Our main results do not rely on the technique used to tune the uncompensated spins: whereas in irradiated AF (as in diluted AF), the surplus magnetization is linked to artificially introduced defects, this pinned component may be associated with such natural structural imperfections as grain boundaries in standard F/AF bilayers. Finally, we conclude that the irreversible uncompensated AF spins (wherever they are pinned) drive the exchange bias field.

The stay of L.C.S. at Orsay was financially supported by $\mathrm{CNPq}$ /Brazil. B.H. acknowledges support by CNRS for a sabbatical stay in Orsay. Partial support by the Deutsche Forschungsgemeinschaft is gratefully acknowledged. T.M. acknowledges support by the Studiens- tiftung des deutschen Volkes.

* On leave from Centro Brasileiro de Pesquisas Fisicas Rua Dr. Xavier Sigaud, 150 Urca, Rio de Janeiro, RJ 22.290180

$\dagger$ Electronic address: mougin@lps.u-psud.fr

[1] W. H. Meiklejohn and C. P. Bean, Phys. Rev. 102, 1413 (1956).

[2] W. H. Meiklejohn and C. P. Bean, Phys. Rev. 105, 904 (1957).

[3] J. Noguès and I. K. Schuller, J. Magn. Magn. Mater. 192, 203 (1999).

[4] A. E. Berkowitz and K. Takano, J. Magn. Magn. Mater. 200, 552 (1999).

[5] R. L. Stamps, J. Phys. D: Appl. Phys. 33, R247 (2000).

[6] D. Mauri, H. C. Siegmann, P. S. Bagus, and E. Kay, J. Appl. Phys. 62, 3047 (1987).

[7] A. P. Malozemoff, Phys. Rev. B 35, 3679 (1987).

[8] T. C. Schulthess and W. H. Butler, J. Appl. Phys. 85, 5510 (1999).

[9] U. Nowak, A. Misra, and K. D. Usadel, J. Magn. Magn. Mater. 240, 243 (2002).

[10] U. Nowak, K. D. Usadel, J. Keller, P. Miltenyi, B. Beschoten, and G. Güntherodt, Phys. Rev. B 66, 014430+0014431 (2002).

[11] W. J. Antel, J. F. Perjeru, and G. R. Harp, Phys. Rev. Lett. 83, 1439 (1999).

[12] J. Noguès, C. Leighton, and I. Schuller, Phys. Rev. B 61, 1315 (2000).

[13] K. H. Bennemann, Non linear optics in metals (Clarendon Press Oxford, 1998), chap. 2 and 3.

[14] H. A. Wierenga, W. de Jong, M. J. Prins, T. Rasing, R. Vollmer, A. Kirilyuk, H. Schwabe, and J. Kirschner, Phys. Rev. Lett. 74, 1462 (1995).

[15] A. Mougin, T. Mewes, R. Lopusnik, J. Fassbender, B. Hillebrands, M. Jung, D. Engel, A. Ehresmann, and H. Schmoranzer, IEEE Transactions on Magnetics 36, 2647 (2000).

[16] A. Mougin, T. Mewes, M. Jung, D. Engel, A. Ehresmann, H. Schmoranzer, J. Fassbender, and B. Hillebrands, Phys. Rev. B 63, 060409R (2001).

[17] J. F. Ziegler, J. P. Biersack, and U. Littmark, The Stopping and Range of Ions in Solids (Pergamon, New York, Oxford, http://www.srim.org/, 1985).

[18] J. Hohlfeld, E. Matthias, R. Knorren, and K. H. Bennemann, Phys. Rev. Lett. 78, 4861 (1997).

[19] Experimentally, see Fig. 1 b b were the magnetic signal is $1.5 \%$ of the average $\mathrm{SH}$ signal in transverse.

[20] J. Güdde, U. Conrad, V. Jähnke, and E. Matthias, Phys. Rev. B 59, 6608 (1999).

[21] The tensor elements entering $\left|\chi_{n m}\right|_{T}$ obtained from Fig. 1 $1 \mathrm{~b}$ differ from those entering $\left|\chi_{n m}\right|_{L}$ but their dose dependence must be the same. 